\title{
Surveillance and Protection-Based Approach for Link Failures over Fiber-to the-Home (FTTH) with Combination of ACS and SANTAD
}

\author{
${ }^{1}$ Aswir Premadi, ${ }^{2}$ Boonchuan Ng \& ${ }^{2}$ Mohammad Syuhaimi Ab-Rahman \\ ${ }^{1}$ Department of Electrical Engineering \\ Institut Teknologi Padang \\ Jl. Gajah Mada, Nanggalo, Padang, 25143, Indonesia \\ ${ }^{2}$ Spectrum Technology Research Division \\ Computer and Network Security Research Group \\ Department of Electrical, Electronics and Systems Engineering \\ Faculty of Engineering and Built Environment \\ Universiti Kebangsaan Malaysia \\ 43600 UKM Bangi, Selangor, Malaysia
}

\begin{abstract}
This paper introduces an in-service transmission surveillance and protection-based approach for fiber failures/faults over fiber-to-the-home passive optical network (FTTH-PON) with an excellent combination of Access Control System (ACS) and Smart Access Network Testing, Analyzing and Database (SANTAD). Our hardware design works on a standard local area network (LAN) using a specially designed hardware interfaced with a microcontroller integrated Ethernet to monitor the status of optical signals flow and provide the restoration against fiber failures/faults in FTTH-PON. We also introduce the centralized management and access control program by means of SANTAD. ACS is used to control the troubleshooting mechanism carried out by SANTAD. This design will be implemented at central office (CO) for distant monitoring and remote controlling each optical fiber line's status as well as for detecting any failures/faults that occurs in the network system downwardly from $\mathrm{CO}$ towards multiple optical network units (ONUs). The scope of this discussion only highlighted on the monitoring and controlling instead of the restoration scheme.
\end{abstract}

Keywords: Microcontroller integrated Ethernet; centralized management; access control; distant monitoring; remote controlling.

\section{Introduction}

FTTH indicates fiber network connections running from $\mathrm{CO}$ to residences or enterprises for bringing triple play services of high-speed data access, voice, and video to the end-users. There are two fundamental FTTH architectures deployed in today's access networks: point-to-point (P2P), which is commonly referred to as active optical network (AON) or active Ethernet; and point-tomultipoint (P2MP), which is commonly referred to as PON. In the AON 
architecture, a single fiber runs all the way from $\mathrm{CO}$ to the home. However, PON has a single fiber that runs from CO to deep in the network and usually terminates at a splitter cabinet [1]. This architecture is called passive because all intermediate equipment between the $\mathrm{CO}$ and ONUs are passive; it has no active electronics and therefore does not need separate power [2].

The introduction of PON allows the network to transport huge amounts of data and provide communication services that play a very important role in many of our daily social and economical activities. Network reliability is an issue of deep concern to network operators being eager to deploy high-capacity fiber networks, since a single failure in the network could result in significant losses of revenue. The importance of network reliability will keep pace with the steadily increasing network capacity. For very-high-capacity future optical networks, carrying multitudes of $10 \mathrm{Gbps}$ channels per fiber strand, a failure of optical connection will interrupt a vast amount of services running on-line, making the connection availability a factor of great significance [3].

Optical fiber maintenance is one of the crucial aspects in network management to enhance the network reliability. Communication networks can be subject to both unintentional failures, caused by natural disasters, wear out and overload, software bugs, human errors, etc., and intentional interruptions due to maintenance. As core communication networks also play a vital military role, key telecommunication nodes were favored targets during the Gulf War, and could become a likely target for terrorist activity. For business customers, disruption of communication can suspend critical operations, which may cause a significant loss of revenue, to be reclaimed from the telecommunications provider. In fact, availability agreements now form an important component of Service Level Agreements (SLAs) between network service providers and customers. In the cutthroat world of modern telecommunications, network operators need a reliable and maintainable network in order to hold a leading edge over the competition [3].

Monitoring equipment for detecting breaks in optical fiber networks is traditionally set up on dark fiber as this provides good balance between material cost, system provisioning effort, and fault detection success rate. Most monitoring systems used for physical fault detection and positioning employ optical time domain reflectometer (OTDR) [4]. OTDR was first reported in 1976 as a telecommunications application and became an established technique for attenuation monitoring and fault location in optical fiber network within the telecommunications industry [5]. OTDR is a well-known means of testing an optical fiber cable assembly in optical networks. The OTDR launches a very narrow pulse into the fiber and then records the response of the cable/connector assembly to this pulse. Both reflections and absorption can be observed in the 
cable, providing the trouble-shooter with the information needed to diagnose cable problems [6]. According to Chomycz [7], OTDR testing is the best method for determining the exact location of broken optical fiber in an installed optical fiber cable when the cable jacket is not visibly damaged. It determines the loss due to individual splice, connector or other single point anomalies installed in a system. It also provides the best representation of overall fiber integrity.

Time period for the OTDR trace results from Rayleigh back scattering (RBS) as the laser source travels along the fiber section of optical fiber are interrupted by an abrupt shifts named as point defects. A point defect is a temporary or permanent local deviation of the OTDR signal either in upward or downward direction. Point defect is caused by a connection, splice or failure along an optical fiber. The output pulse is indicated as end of an optical fiber from Fresnel reflection occurring at each fiber end. Fiber point defects occur from splicing or bending during the fiber's plant installation. Point defect that occurs at a fiber joint is easy to identify if the location of a fiber joint is generally known. Normally, a reflective or non-reflective fault occurs at a fiber joint location. In most circumstances, an optical connector produces a reflective fault, while an optical splice produces a non-reflective fault. However, reflective and non-reflective faults that occur at other than fiber joint is identified as a fiber break, crack or bend. A fiber break produces a reflective fault, while fiber cracks and bends produce a non-reflective fault [8].

One problem encountered in most OTDR surveillance systems is the OTDR test conducted from the out-of-service ONU (in upstream direction). However, this approach would require a lot of time and effort. Moreover, OTDR can only display a measurement result one line at a time. Therefore, this makes the conventional OTDR's measurement analysis task in PON fiber plant with a large number of subscribers and large coverage area become very difficult [9]. One common approach to alleviate this problem is to emit the OTDR pulse from $\mathrm{CO}$ to ONUs (in downstream direction). When many distribution fibers are being tested from $\mathrm{CO}$ toward the optical splitter/coupler and all the way to ONUs, the reflection of each of the different fibers will be combined (added into one trace as summarized in Figure 1) and the interpretation of the trace OTDR will become more difficult and often not possible [10].

Some researchers had discussed about the monitoring issues with OTDR and recommended a number of possible methods $[11,12,13]$ to overcome these problems (i.e., achieve desired network survivability). These approaches can monitor the network system without affecting other in-service channels. However, these methods need relatively expensive additional sources or devices that impose high-maintenance cost since the network service providers need to 
keep capital and operational expenditures (CAPEX and OPEX) low in order to be able to offer economical solutions for the customers. Therefore, improving network reliability performance by adding redundant components and systems have shortcomings in terms of implementation cost and flexibility [12]. In addition, these methods are complex and difficult to implement that has prohibited them as a practical solution [14].

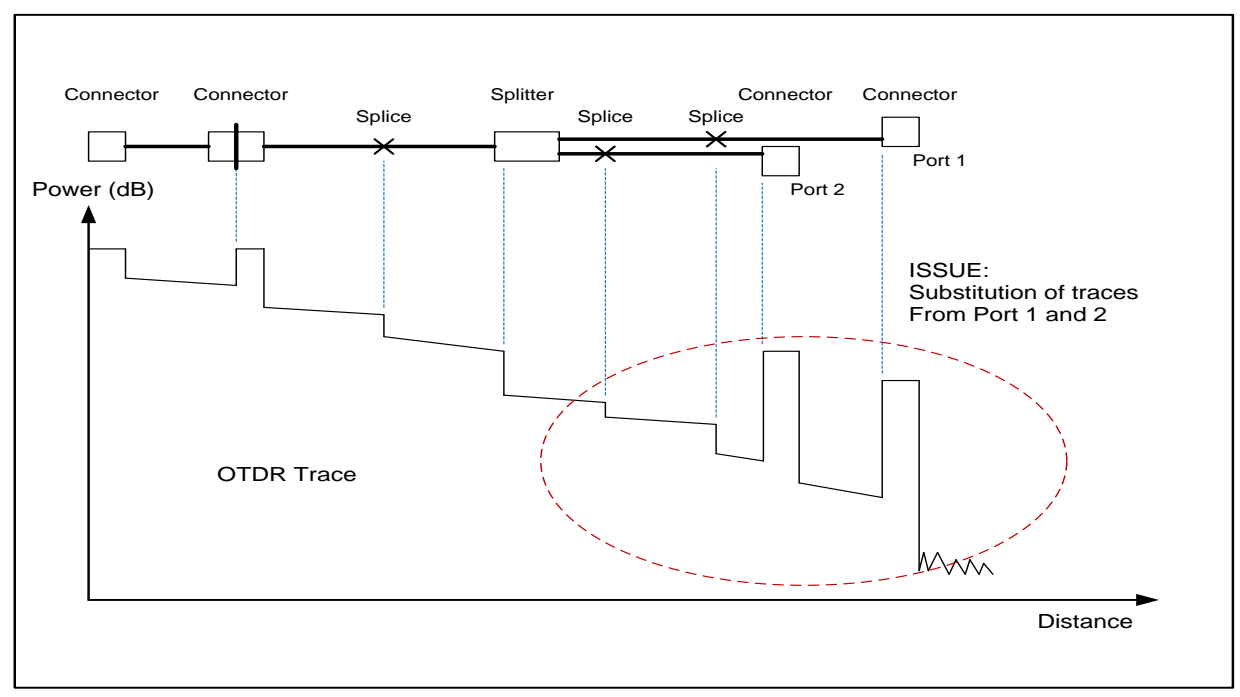

Figure 1 Critical issues for OTDR testing and characterization measurement on a conventional PON containing optical splitter/coupler using in downstream direction.

\section{Access Control System (ACS)}

In this study, we propose and demonstrate a novel survivability FTTH-PON based on ACS with the objective to reduce cost and misspend time. ACS is a functional tool for monitoring, testing, and analyzing as well as activating the protection switch in the restoration process for FTTH-PON network system. ACS is the core of proposed design. It is located at the middle of the network system for controlling the devices/components in feeder region and drop region. It becomes an intelligent control centre used as an intermediate medium for controlling the monitoring and protection system in the access network. The system design consists of a microcontroller system, In-Line Monitoring (ILM), and Restoration Scheme Activate (RSA).

ACS controls the status of any optical switch device connected to it and transmits its status to the PIC18F97J60 microcontroller. Its then arranges the 
information in the form of a packet and transmits it over the LAN using the embedded Ethernet system. Generally, the hardware development of ACS is integrated in a single system, which also includes $1 \times 8$ optical switch, passive optical splitter, OTDR, and remote personal computer (PC). Tapping 3\% of the downstream and upstream signal by using coupler can recognize the status of feeder section and drop section. If breakdown occurs in feeder section, ACS will send a signal to activate the dedicated protection scheme. But if the breakdown is detected in drop section, ACS will recognize the related access line by $3 \%$ tapped signal that is connected to every access line. The activation signal is then sent to activate the dedicated protection scheme. However, if fault is still not restored, the shared protection scheme will be activated. The monitoring signal section is responsible for sensing fault and its location whereas generation of signal activation is sent by activation section in ACS [15].

An optical switch can be used to monitoring and measurement. The device has optimized mechanical latching system combined with a prism, and used in specialized systems such as optical network. This type of switch enables signals in optical fiber or integrated optical circuits to be selectively switched from one circuit to another, or may be used for alternate routing of an optical transmission path, e.g., routing around a fault [16].

As technology advances, PIC microcontroller is manufactured by Microchip Technology Inc. Currently, this is one of the most popular microcontroller uses in many applications. It is a self-contained computer-on-a-chip that integrates a microprocessor, input and output ports, memory for program and data storage, an internal clock, and one or more peripheral devices such as timers, counters, analog-to-digital converters (ADCs), communication facilities, watchdog circuits and special hardware features. Among all the PIC microcontroller families, especially the PIC18F97J60 of devices feature an embedded Ethernet controller module. It was integrated Media Access Control (MAC) and 10BaseT PHY, making Ethernet communication possible.

The 8-bits microcontroller has enjoyed a tremendous growth in embedded systems applications. It is a fine chip that is easy to program by means of a simple device attached to the parallel or serial port or Ethernet port. With the $128 \mathrm{~Kb}$ of code space available on the PIC18F97J60, a Transmission Control Protocol/Internet Protocol (TCP/IP) stack can easily be accommodated while leaving plenty of program memory for the application, which is one of the important factors in such a system $[17,18]$. It is chosen due to its variety of hardware modules needed for survivability in FTTH-PON. 


\section{Smart Access Network - Testing, Analyzing and Database (SANTAD)}

SANTAD is a centralized access control and surveillance system that enables the network operators and field engineers to view traffic flow and detect breakdown as well as other circumstances that may require some appropriate action with the graphical user interface (GUI) processing capabilities of Microsoft Visual Basic 2008 (VB9) programming. SANTAD increases the workforce productivity and facilitates the network management of network through centralized monitoring and troubleshooting from CO. The system accurately detects and locates fiber degradations/failures, and alerts the field engineers with the details of failures/faults within 30 seconds. The system database allows the network operators and field engineers to assess long term network performance.

The functionalities of SANTAD can be generally classified into preventive maintenance and post-fault maintenance, which can assist the network operators and field engineers to perform more efficiently the following activities (for managing the PON network system):

- Record Events/data

- Control/monitor information for preventive maintenance

- Present surveillance image (visual feedback)

- Provide a control function to intercom all subscribers with CO

- Monitor and control the network performance

- Detect degradations before a fiber fault occurs for preventive maintenance

- Detect any fiber failure/fault that occurs in the network system and troubleshoot it for post-fault maintenance

The working principles of SANTAD are divided into three main parts: (i) Network testing and troubleshooting with OTDR, (ii) Interfacing OTDR test module with remote personal computer (PC)/laptop, and (iii) Centralized monitoring and advanced data analyzing. A commercially available OTDR is located at $\mathrm{CO}$ for network testing and troubleshooting in downstream direction (from CO towards customer sides). Due to the U-band (ultra long wavelength band; $1625-1675 \mathrm{~nm}$ ) light is different from the wide communication band (1260-1600 nm) and has been reserved for standard PON monitoring, the network system can perform in-service testing by using $1625 \mathrm{~nm}$ light source with no degradation to the transmission quality and interruption. A tapper circuit is designed to allow the OTDR testing signal to bypass the optical splitter in a conventional PON when emitted in downstream direction. 
The OTDR is accomplished through the 10/100 Ethernet port that is connected to a remote PC/laptop. The Ethernet remote interface allows the users to access (connect) the OTDR test module over any Internet-connected PC via standard commands for programmable instruments (SCPI) according to IEEE 488 standard. This enable the users to run and operate the OTDR test module from a remote $\mathrm{PC} /$ laptop at $\mathrm{CO}$, point of link control (remote site), or from anywhere in the world without on-site personnel for distant monitoring. All the OTDR measurements are accumulated to be displayed on one screen for centralized monitoring and advanced data analyzing.

SANTAD is focusing on providing survivability through event identification against losses and failures. SANTAD involves the fiber fault detection, notification, verification, and restoration functions. Under normal working condition, it allows the network services providers and operators to determine the path used by the services through the network, whereas under failure conditions, it allows the fields engineers to identify the faulty fiber and failure location without making a site visit. SANTAD enables the network service providers and field engineers to analyze the optical fiber line's status, display the line's detail, track the optical signal level, and losses as well as monitor the network performance. In combination of the distinctive features, SANTAD provides a convenient way to solve the particular upwardly or downwardly measuring issues with OTDR and produce capability of fiber fault localization in an optical access network.

SANTAD will be designed to operate by itself with a minimum need for operator actions. SANTAD ensures that when detecting a fiber failure/fault on the primary entity of the FTTH-PON network system, it is automatically reports the failure status to the field engineers and technicians, and then the field engineers and technicians can determine sharply the break point before taking some appropriate actions. Meanwhile, they activate the restoration scheme to switch the traffic from failure line to protection line to ensure the traffic flows continuously. This functionality alerts the service providers and field engineers of fiber degradation or failure/faults before it is reported by the customer premises or subscribers.

SANTAD can potentially improve the survivability and increase the monitoring capabilities in FTTH-PON as well as can overcome the upwardly or downwardly monitoring issues with conventional fiber fault localization technique by using OTDR. Overall, it can reduce the time needed to restore the fiber fault to maintain and operate the FTTH more efficiently. 


\section{Optical Network Monitoring and Management}

We have experimentally demonstrated the proposed survivability scheme through optical switch based on microcontroller in a FTTH-PON. Figure 2 briefly explains the entire work. Each ONU is connected to optical splitter output terminal by two fibers: working line and protection line through optical switch that is controlled by ACS. ACS is focusing on providing survivability through the RSA against failures/faults by means of dedicated and shared protection that is applied in FTTH-PON. In this design, ACS is used to monitor the status of both working line and protection line. ACS recognizes all type of failures and sends the activation signal to the related optical switch according to the activated protection mechanisms.

In order to locate a failure without affecting the transmission services to other customers, it is essential to use a wavelength different from the triple-play services operating wavelengths (optical signals; $1310 \mathrm{~nm}, 1490 \mathrm{~nm}$, and 1550 $\mathrm{nm})$ for failure detection. ACS integrated Ethernet is using the $1625 \mathrm{~nm}$ testing signal for failure detection control and in-service troubleshooting. The tripleplay signals are multiplexed with $1625 \mathrm{~nm}$ OTDR testing signal. The OTDR is located at $\mathrm{CO}$ and connected to a remote personal computer (PC) to display all the troubleshooting results. The principal limitation to live fiber monitoring at $1625 \mathrm{~nm}$ will come from the spontaneous Raman scattering noise that reaches the OTDR port. In case of bidirectional transmission, OTDR power and transmission power levels may require adjustments so that effects remains negligible.

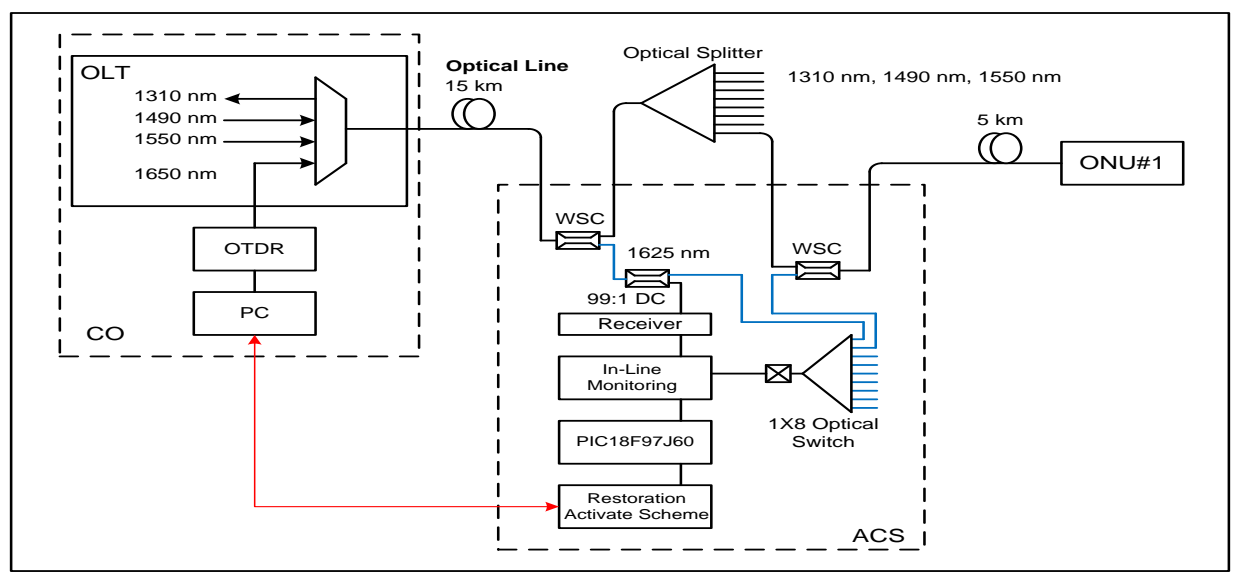

Figure 2 Schematic diagram of the proposed surveillance and protection-based approach for link failures over FTTH-PON with an excellent combination of ACS and SANTAD. 
As illustrated in Figure 2, the triple-play signals are multiplexed (combined) with $1625 \mathrm{~nm}$ OTDR testing signal. A tapper circuit is designed to allow the OTDR testing signal to bypass the optical splitter in a conventional PON when emitted in downstream direction (from $\mathrm{CO}$ towards multiple customer residential locations). When four kinds of signals are distributed, the 1625 testing signal will be split up by the wavelength selective coupler (WSC), which is installed before the optical splitter. The WSC coupler only allows the 1625 $\mathrm{nm}$ signal to enter into the tapper circuit and filter all unwanted signals that contaminate the OTDR measurement. The downstream signal will go through the WSC, which in turn connected to the optical splitter before it reaches the multiple ONUs at different customer residential locations. On the other hand, the $1625 \mathrm{~nm}$ signal, which is demultiplexed by WSC coupler, will be split up again in power ratio $99: 1$ by using directional coupler (DC) to activate the microprocessor system. The $99 \%$ of $1625 \mathrm{~nm}$ signal will then be configured by using optical splitter whose outputs are connected to single lines of ONU. The operational of optical switch is controlled by ACS that is activated by $1 \%$ of $1625 \mathrm{~nm}$ testing signal [19].

In order to enable wavelength splitting (de-multiplexing) and combining (multiplexing) in the tapper circuit, WSC coupler designed for the optical signals, which have different light wavelengths, can be separated or combined to transmit in single optical fiber as shown in Figure 3. The WSC coupler is designed on silica substrate with compliance of FTTH-PON wavelengths. The WSC coupler is used as a router for specific wavelength in order to detect any optical line failure in FTTH-PON application. The triple-play signals enter the waveguide in port 1 and OTDR testing signal $(1625 \mathrm{~nm})$ enters the waveguide at port 3. The $1625 \mathrm{~nm}$ testing signal generated by the OTDR will be used to scan the status of FTTH-PON. All the wavelengths must flow out through port 2. In reverse mode, the device is applicable to split the $1625 \mathrm{~nm}$ testing signal from triple-play signals [20].

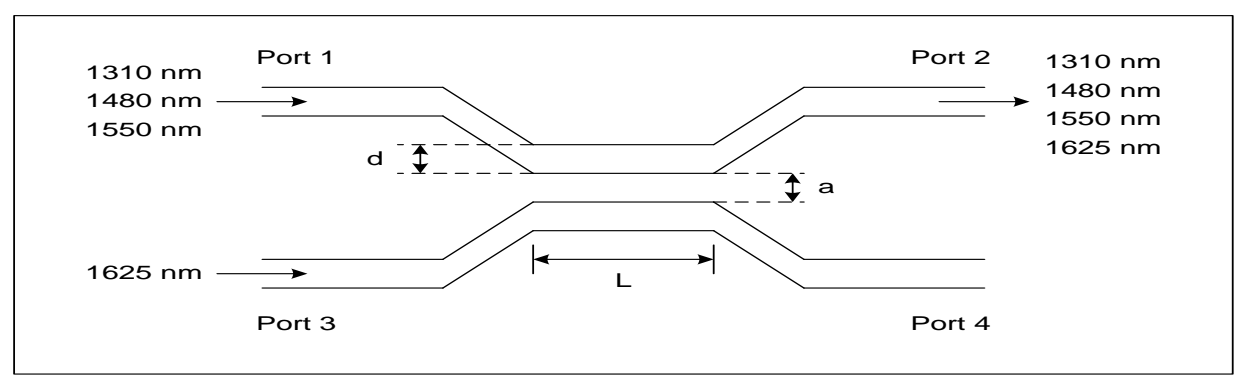

Figure 3 Structure of WSC which operate the wavelength used in FTTH-PON application. 


\section{$5 \quad$ Results and Discussions}

The ACS included the Ethernet Module System is designed to transmit the status of optical switch device connected to our system over the LAN or Internet connectivity applications through Ethernet connection. The status of each line is then automatically updated to a web server that can be accessed by the operator network via internet or LAN. ACS sends three bit signal to activate the optical switch for scanning each optical fiber line, and sends or receives one ready signal to indicate each working line. When any failure occurs in the working line, the first optical switch will route the disturbed signals to the local protection line and the second optical switch will be activated simultaneously to switch the signals back to the transmission line. Table 1 presents the entire optical switch input/output of the proposed live fiber monitoring in ACS scheme. Therefore, ACS can detect line failures by monitoring each optical switch routing.

When the PIC 18F97J60 gets an ILM request, it checks all optical switch lines attached to the ACS. After scanning the ports of the microcontroller, a string of 1 's and 0 's is formed, depending upon the state of the optical switch connected to each ACS. 1 stands for high and 0 stands for low.

Table 1 I/O Optical Switch.

\begin{tabular}{|c|c|c|c|c|c|}
\hline \multirow{2}{*}{$\begin{array}{l}\text { Route } \\
\text { Optical } \\
\text { Switch }\end{array}$} & \multicolumn{3}{|c|}{$\begin{array}{c}\text { Input } \\
\text { Terminal } \\
\end{array}$} & \multirow{2}{*}{$\begin{array}{c}3 \text { bit } \\
\text { Command }\end{array}$} & \multirow{2}{*}{$\begin{array}{l}\text { Output } \\
\text { Ready } \\
\text { (RDY) }\end{array}$} \\
\hline & D0 & D1 & D2 & & \\
\hline Line 1 & $\mathrm{~L}$ & $\mathrm{~L}$ & $\mathrm{~L}$ & 000 & 1 \\
\hline Line 2 & $\mathrm{~L}$ & $\mathrm{~L}$ & $\mathrm{H}$ & 001 & 1 \\
\hline Line 3 & $\mathrm{~L}$ & $\mathrm{H}$ & $\mathrm{L}$ & 010 & 1 \\
\hline Line 4 & $\mathrm{~L}$ & $\mathrm{H}$ & $\mathrm{H}$ & 011 & 1 \\
\hline Line 5 & $\mathrm{H}$ & $\mathrm{L}$ & $\mathrm{L}$ & 100 & 1 \\
\hline Line 6 & $\mathrm{H}$ & $\mathrm{L}$ & $\mathrm{H}$ & 101 & 1 \\
\hline Line 7 & $\mathrm{H}$ & $\mathrm{H}$ & $\mathrm{L}$ & 110 & 1 \\
\hline Line 8 & $\mathrm{H}$ & $\mathrm{H}$ & $\mathrm{H}$ & 111 & 1 \\
\hline
\end{tabular}

We have experimentally demonstrated our proposed scheme. As shown in Figure 4, the switching of fiber lines between the eight wavelengths can be clearly observed. 


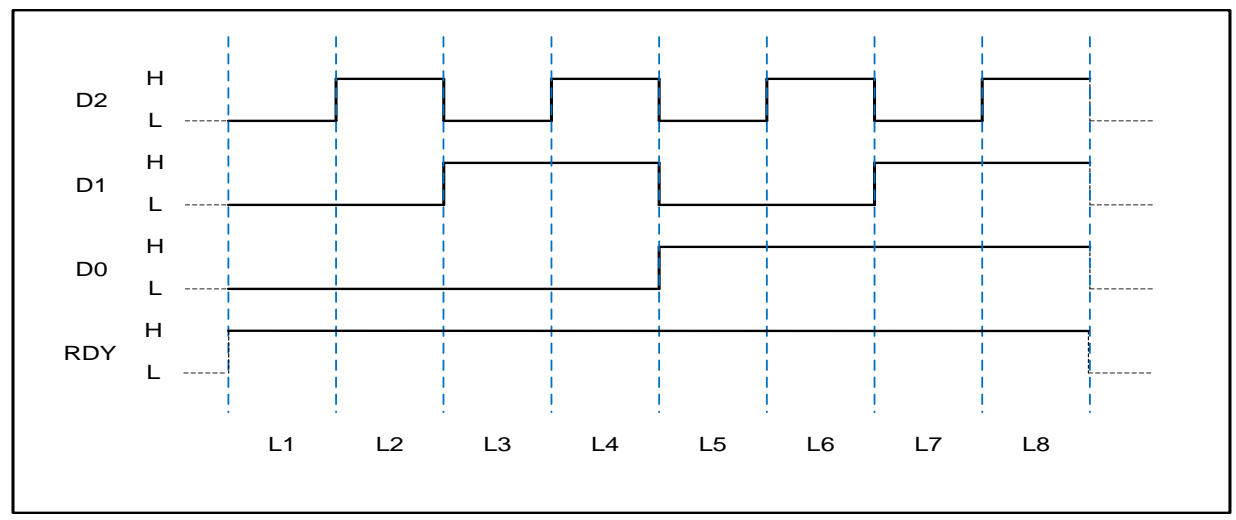

Figure 4 Curve of optical power at eight switch output.

To simulate the fault identification process, Figure 5 shows the optical power output of the proposed fiber line monitoring when fiber lines 5 and 8 are broken by an external force application. The solid line indicates normal operation from fiber lines 1-4, 6, \&7, and the dotted line shows fiber fault behavior when fiber lines $5 \& 8$ are disconnected, and the output ready is low or 0 , as shown in Table 2. The experimental results show that our proposed scheme is feasible.

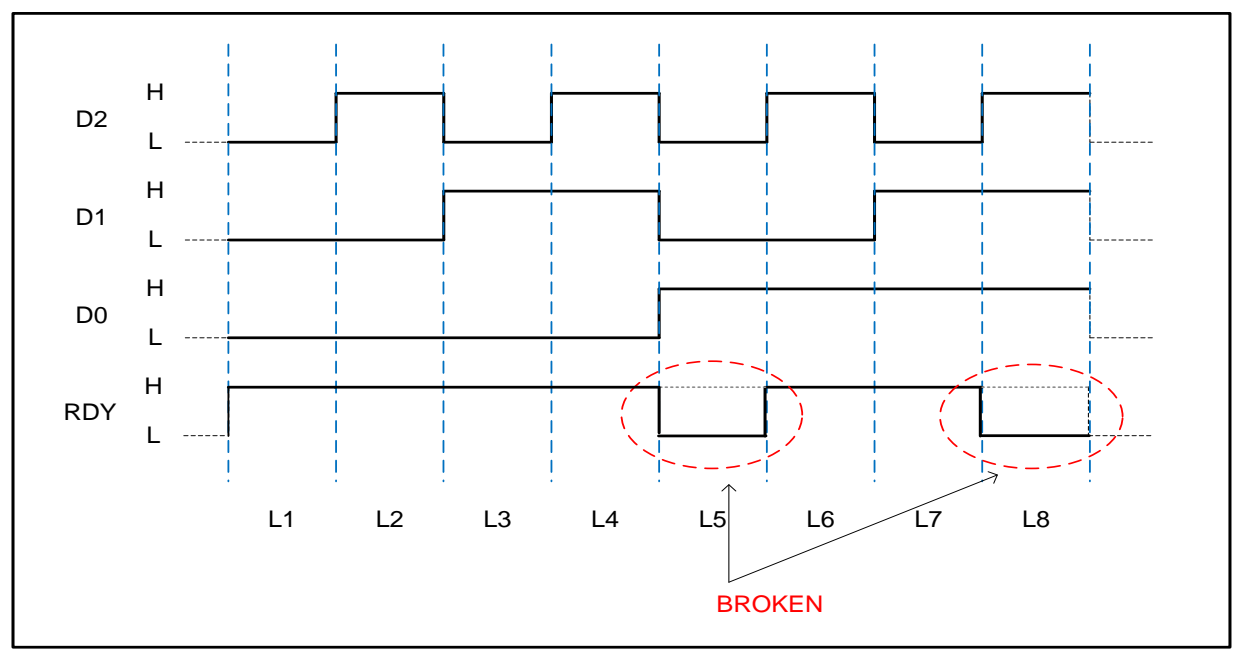

Figure 5 Optical power output of the proposed monitoring when the fiber line 5 and 8 are broken by external force applied. 
Surveillance and Protection-Based Approach for Link Failures 45

Table 2 I/O Optical Switch when fiber line 5 and 8 are broken.

\begin{tabular}{cccccc}
\hline $\begin{array}{c}\text { Route Optical } \\
\text { Switch }\end{array}$ & \multicolumn{3}{c}{$\begin{array}{c}\text { Input } \\
\text { Terminal }\end{array}$} & $\begin{array}{c}\text { 3 bit } \\
\text { Command }\end{array}$ & $\begin{array}{c}\text { Output Ready } \\
\text { (RDY) }\end{array}$ \\
\cline { 2 - 5 } & D0 & D1 & D2 & & 1 \\
Line 1 & L & L & L & 000 & 1 \\
Line 2 & L & L & H & 001 & 1 \\
Line 3 & L & H & L & 010 & 1 \\
Line 4 & L & H & H & 011 & 1 \\
Line 5 & H & L & L & 100 & 0 \\
Line 6 & H & L & H & 101 & 1 \\
Line 7 & H & H & L & 110 & 1 \\
Line 8 & H & H & H & 111 & $\mathbf{0}$ \\
\hline
\end{tabular}

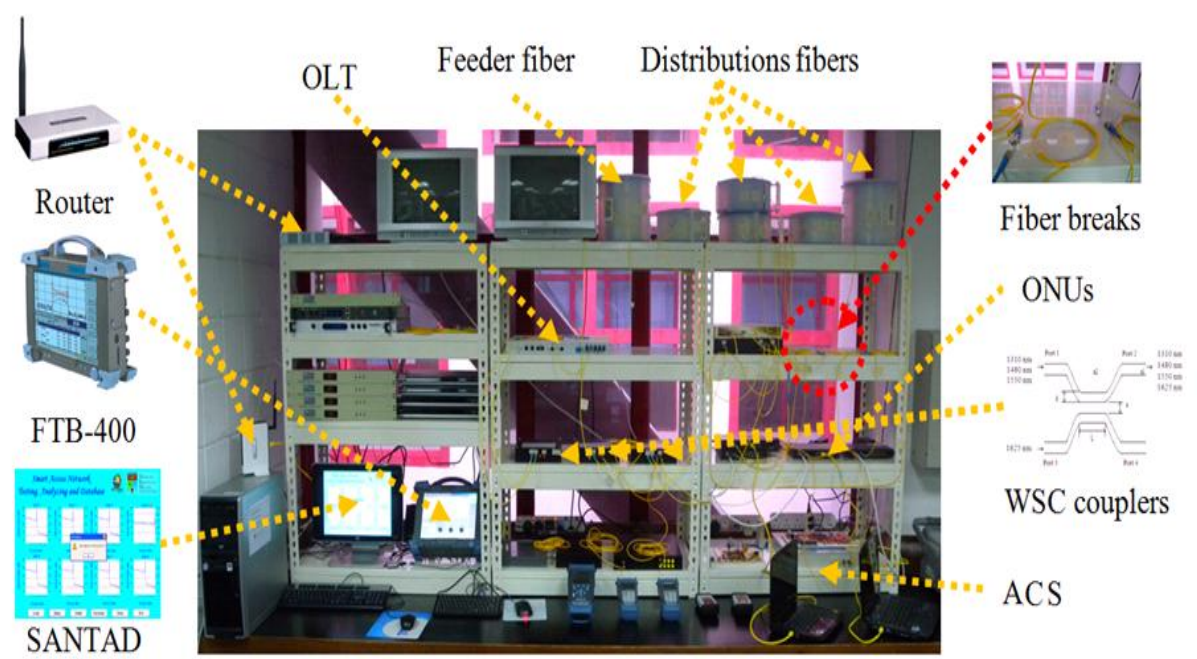

Figure 6 Photographic view for network monitoring and troubleshooting with combination of ACS and SANTAD.

The lab prototype of SANTAD is implemented in a PON network test-bed composed by $20 \mathrm{~km}$ fiber for monitoring and analyzing the network performance. The length of feeder fiber is $15 \mathrm{~km}$. The fiber link in distribution region between the optical splitter and each ONU is about $15 \mathrm{~km}$. In normal operation, both the upstream and downstream signals travel through a 
transmission distance of $20 \mathrm{~km}$ from OLT towards each ONU. The network test-bed is set-up to serve as a platform to study the mechanisms and characteristics of optical signal in normal operation (good condition) and failure condition (breakdown).

To verify the concept and benefits of SANTAD, we conduct an experiment for evaluating our in-service fault localization methodology in our proposed design. Here we are specially focusing on identifying the link failures in the network system. The instruments and measurement equipments used in the experiment are summarized in Figure 6. During the observation, we disconnected the fiber connector to represent a fiber failure/fault at the corresponding position. It visualizes the actual break point of an optical line at that distance in a real condition.

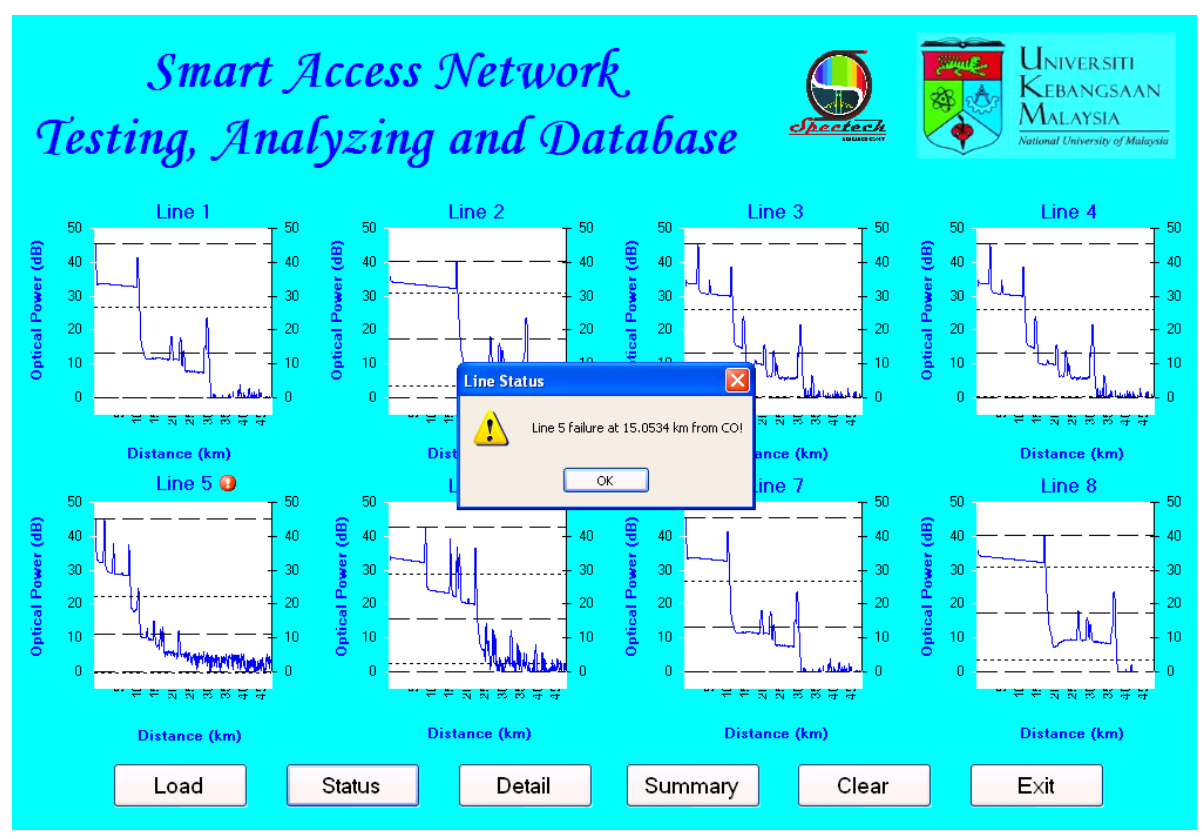

Figure 7 Execution display in Centralized Monitoring form.

Our in-service fault localization results are presented in Figures 7 and 8. Every eight network testing results will be displayed in Centralized Monitoring form for centralized monitoring and advanced analysis, where the distance $(\mathrm{km})$ is represented by the $\mathrm{x}$-axis and optical signal level $(\mathrm{dB})$ is represented by $\mathrm{y}$-axis. A failure message "Line x FAILURE at $z \mathrm{~km}$ from CO!" will be displayed to 
inform the field engineers if SANTAD detects any fiber fault in the network system.

Figure 7 depicts the capability of SANTAD to configure the optical signal level and attenuation/losses through event identification method. The failure location is identified by a drastic drop of optical power level among a number of optical fiber lines in a FTTH-PON. To obtain further details on the performance of specific line in the network, every measurement results obtained from the network testing are analyzed in the Line's Detail form as illustrated in Figure 8. SANTAD is able to identify and present the parameters of each optical fiber line such as the line's status, attenuations, reflection and return losses, distance, failure location, and other details as shown in the OTDR's screen. The analysis results will then be stored in database to allow the network operators and field engineers assessing long term network performance (see Figure 9).

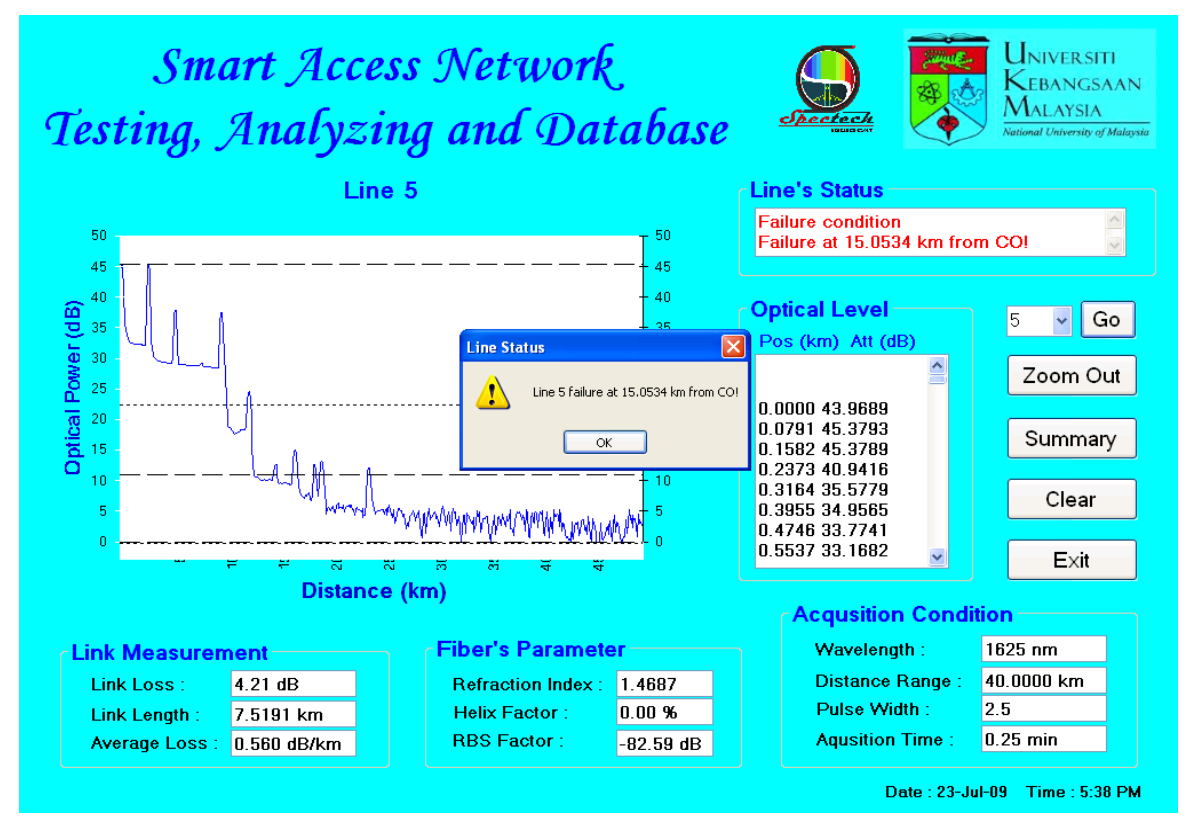

Figure 8 Execution display in Line's Detail form. 


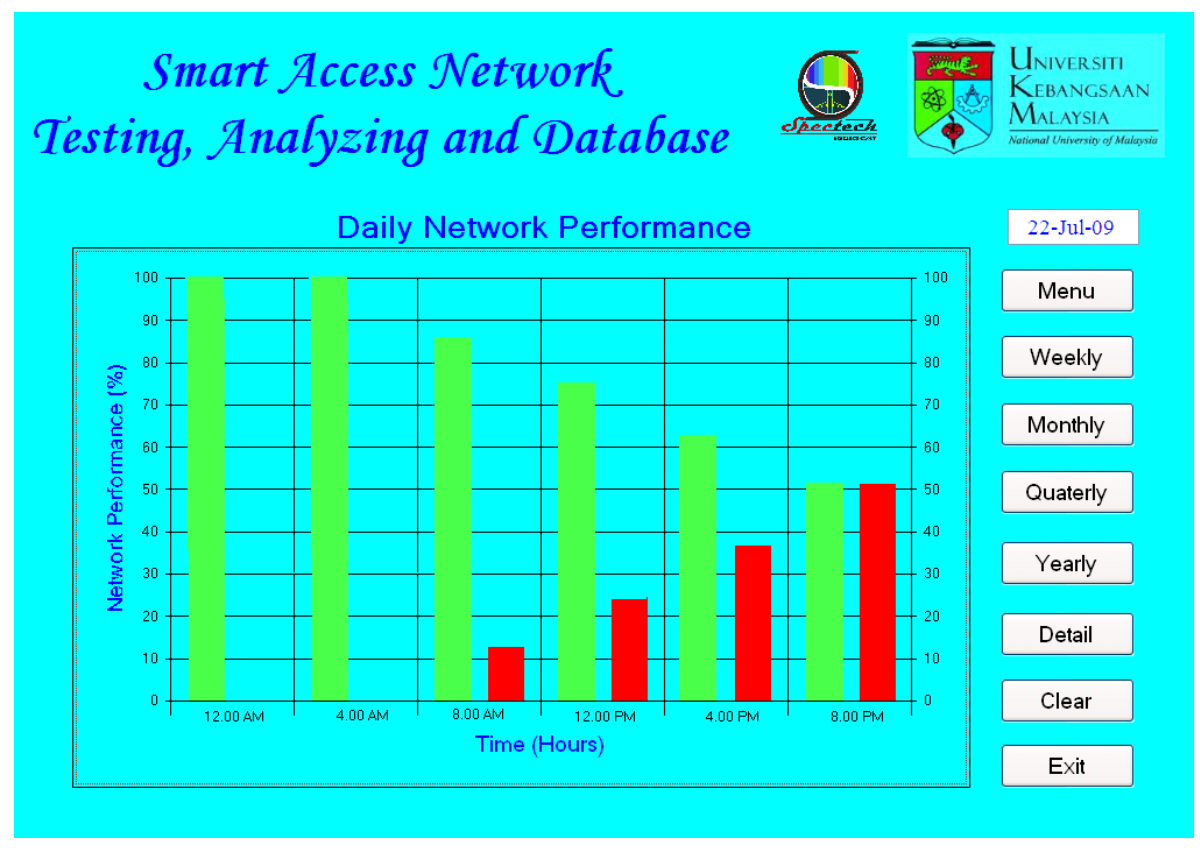

Figure 9 Analysis of the relationship between network failure rate and network.

\section{Summary and Future Work}

A survivability of a PON monitoring with microcontroller has been proposed in this paper. With an excellent combination of hardware (ACS) and software (SANTAD) development, we have successfully developed a lost-cost surveillance and protection system for PON appliances. The experimental results show that the developed system is able to identify the status of each fiber link without affecting the delivery of triple-play services. By using the available specification, the monitoring process can be remotely accessed 24 hours a day and 7 days a week. When problems are detected and diagnosed, the system helps minimize network downtime, rapidly restore failed services, and efficiently manage network performance. In the future, our research team aim continues to work at diminishing the impact on traffic and on the quality of the measurements so that the deployment of live fiber monitoring can be readily available and applicable to most situations.

\section{References}

[1] Hutcheson, L., FTTx: Current Status and the Future", IEEE Commun. Mag. 46(7), 136-145, 2008. 
[2] Occam Networks, FTTx: selecting the best architecture for the access network. $2005 . \quad$ Available: http://www.convergedigest.com/whitepapers/documents/Occam\%20FTT X\%20white\%20paper.pdf.

[3] Wosinska, L., Colle, D., Demeester, P., Katrinis, K., Lackovic, M., Lapcevic, O., Chapter 9: network resilience in future optical networks, Towards Digital Optical Networks, Springer Berlin / Heidelberg, pp. 253284, 2009.

[4] Chen, H., Leblanc, M., Plomteux, O., Live-Fiber OTDR Testing: Traffic and Measurement Impairments, EXFO Electro-Optical Engineering Inc., pp.1-7, 2007.

[5] King, D., Lyons W. B., Flanagan, C., Lewis, E., Interpreting Complex Data from a Three-Sensor Multipoint Optical Fiber Ethanol Concentration Sensor System using Artificial Neural Network Pattern Recognition, Measurement Science and Technology, 15, 1560-1567, 2004.

[6] Harres, D. N., Built-in Test for Fiber Optic Networks Enabled by OTDR, In Proceeding of $25^{\text {th }}$ Digital Avionics Systems Conference, IEEE, Portland, pp. 5A1-5A8, 2006.

[7] Chomycz, B., Fiber Optic Installation: A Practical Guide, New York: McGraw-Hill, 1996.

[8] Integrated Publishing, Optical Time Domain Reflectometry, 2008, Available: http://www.tpub.com/neets/tm/109-12.htm.

[9] Network Infrastructure Committee. FTTH Infrastructure Components and Deployment Methods, 2007, Available: http://www.europeftthcouncil.com.

[10] Anderson, D.R., Johnson, L., and Bell, F.G., Chapter 10: Analyzing passive networks containing splitters and couplers, Troubleshooting Optical Fiber Networks Understanding and Using Optical Time-Domain Reflectometers, California, US: Academic Press, Elsevier, pp. 279-290, 2004.

[11] Caviglia, F., Biase V.C.D., Optical Maintenance in PONs, In Proceeding of $24^{\text {th }}$ European Conference on Optical Communication (ECOC'98), Madrid, Spain, pp. 621-625, 1998.

[12] Prat, J., Optical networks: towards bandwidth manageability and cost efficiency, 2007, Available: http://www.e-photonone.org/ephotonplus/servlet/Utils.MostrarFitxerPublic?fitxer=D_VDA_3. pdf\&pathRelatiu=EPhoton+One+\%2B\%2FPublic\%2FPublic+Deliverabl es\%2F.

[13] Yuksel, K., Moeyaert, V., Wuilpart, M., Mégret, P., Optical Layer Monitoring in Passive Optical Networks (PONs): a Review, In Proceedings of $10^{\text {th }}$ Anniversary International Conference on Transparent Optical Networks (ICTON 2008), Athens, Greece, pp. 92-98, 2008. 
[14] Lee, L., Kang, S. B., Lim, D. S., Lee, H. K., Sorin, W. V., Fiber Link Loss Monitoring Scheme in Bidirectional WDM Transmission using ASEInjected FP-LD, IEEE Photonics Technology Letters, 18(3), 523-525, 2006.

[15] Rahman, M.S.A., Yusof, M.T.M., Development of Optical Multifunctional Switch, In Proceeding of Wireless and Optical Communication Network (WOCN '08), $5^{\text {th }}$ IFIP International Conference, pp.1-7. 2008.

[16] The Omron Electronic Component Website, 2008 [Online], Available: http://components.omron.eu/.

[17] The Microchip Website, 2008 [Online], Available: http://www.microchip.com.

[18] PIC18F97J60 Family Data Sheet, Microchip Technology Inc., 2008, Doc. no. DS39762D.

[19] Rahman, M. S. A., Ng, B. C., MATLAB-based Graphical User Interface Development for Centralized Failure Detection System (CFDS) in SCAN Network. Journal of Optical Communications (JOC), 29(3), 152-156, 2008.

[20] Rahman, M. S. A., Rashid, A. R. A., Ehsan, A. A., and Shaari, S., The Characterization of FTTH Wavelength Selective Coupler, in Proc. of 2008 IEEE Int. Conf. on Semiconductor Electronics (ICSE 2008), pp. 302-305, 2008. 